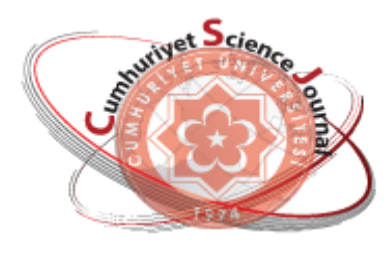

e-ISSN: $2587-246 X$

ISSN: $2587-2680$

\section{Cumburiyet Scionce Journal $\operatorname{csg}$}

Cumhuriyet Sci. J., Vol.40-1(2019) 79-86

\title{
Amplitude of Elastic Scattering for the Region of Large Impact Parameters
}

\author{
Sajida G. ABDULVAHABOVA*(D), N.Sh. BARKHALOVA, T.O. BAYRAMOVA
}

Baku State University, Baku, Azerbaijan

Received: 31.05.2018; Accepted: 07.10.2018

http://dx.doi.org/10.17776/csj.512886

\begin{abstract}
The work is dedicated to consequences of analyticity and unitarity of the scattering amplitude. Using the Gaussian quasipotential an equation for the scattering amplitude matrix is obtained and formula is derived for the cross sections. The dependence of the cross section and ratio of the real part of the amplitude in the forward scattering to its imaginary part of on the momentum are discussed. The steep Gaussian peak for cross section at small angles is followed by the exponential (Orear) regime. Results from theoretical approach are compared with experimental data.
\end{abstract}

Keywords: Quasipotential, scattering amplitude, unitarity, cross section, impact parameter.

\section{Büyük Etki Parametreleri Bölgesi İçin Elastik Saçılma Genliği}

Özet. Bu çalışmada, saçılma genliğinin analitikliğinin ve uniterliğinin gerekliliği incelenmiştir. Gaussiyen yarı-potential kullanılarak, saçılma genliği matrisi için bir denklem elde edilmiş ve tesir kesitleri için bir formül elde edilmiştir. Tesir kesitin bağımlılığı ve ileri saçılmadaki genliğin gerçel bölümünün momentum üzerindeki sanal kısmına oranı tartışılmıştır. Küçük açılarda tesir kesiti için dik Gaussiyen zirveyi üstel (Orear) rejim takip eder. Teorik yaklaşımdan elde edilen sonuçlar deneysel veriler ile karşılaştırılmıştır.

Anahtar Kelimeler: Yarı potansiyel, saçılma genliği, üniterlik, tesir kesiti, etki parametresi.

\section{INTRODUCTION}

A theoretical analysis of the angular distribution of the fragments arising from the break-up of the projectile by the nuclear and interaction with the target is very useful and necessary for the experimental investigations. More useful would be the exclusive experiments where the scattering process of the projectile is separated from the background of other reactions by means of the coincidence detection of the two outgoing fragments together with a simultaneous measurement of their energies [1].

In the theory of diffraction, the reaction is considered as a quantum mechanical process due to the fact that different components of the wave function of the incident hadron have a different probability of interaction with the target [2]. As a result, the wave function is distorted. If we expand it over a complete set of functions, after collision, it contains not only the initial hadron function and other states of the incident particle. In this case we are talking about those components of the wave function for which the probability of interaction with the target is small, i.e. In the course of the collision, only a small part of the target is excited (an elastic collision with only one of its constituent particles). Since other wise the quark wave function of the target will lose its coherence and the 
target will decay into a large number of secondary hadrons. To do this, we must use the "point" component of the wave function of the incident hadron. This is possible in the following cases: quarks and gluons are at a small distance from each other and the scattering cross section is very small; - go into the field of large impact parameters. In particular, has been the touchstone of the theory since its first derivation, showing a remarkable agreement between the predicted and experimental structure of the angular scattering distribution. The analysis of hadron-nucleus cross sections in the difraction approximation can be used to extract information on the scatering amplitude parameters. For this it will be necessary to measure the hadronnucleus cross sections at various incident-hadron energies.

According to the model developed in [3], when the de Broil wavelength of the incident particle is much smaller than the size of the target nucleus, the interaction of each incident particle with the nucleus can be considered as a process of interaction along a narrow tube inside the nucleus, whose distance from

the center of the nucleus is determined by the impact parameter $b$. In this paper, we discuss the cross section of elastic scattering for the region of large impact parameters $b$.

\section{THE AMPLITUDE OF ELASTIC SCATTERING}

We will discuss some aspects of nucleon-nucleon collisions without physical overlap, i.e. collisions with impact parameters, $b$, larger than the sum of the nuclear radii, $R$, i.e. $b>2 R$. Particles can be produced in these collisions through an interaction of the fields of the nuclei. The interactions can involve both the electromagnetic and nuclear fields, but because of the short range of the nuclear force, purely nuclear processes are suppressed for $b>2 R$.

If the momentum transfers from the nuclei are small enough $(\mathrm{q}<\mathrm{kc} / \mathrm{R})$, the fields both nuclei couple coherently to all nucleons. This enhances the cross sections and gives the events a unique signature, which can be used for identification. The restrictions on the momentum transfer do not prevent the production of heavy systems, however, in high-energy collisions.

The scattering of hadrons at high energies is a multiparticle process, as a result of which the unitarity condition reduces to an unsolvable infinite chain of interlocking equations. One of the methods that would allow us to construct an elastic scattering amplitude that satisfies at least the twoparticle unitarity condition is the use of a quasipotential [4].

The quasipotential in space configuration depends on the velocity and is nonlocal. In addition, it depends on the total energy of the system and is a complex function. Choosing a quasipotential in the form of a smooth, local (in configurational space) function that depends on energy, with a positive definite imaginary part, it is possible to correctly describe the basic properties of hadron scattering at small and large angles [5,6].

The potential description of scattering with a given quasipotential permits a description region of the phase shifts of individual iteration which are essential in the transition from the description region of momentum transfer to the Orear region. The probability description can be considered as a justification for the introduction of smooth quasipotentials into field theory, and in addition it appears to be more promising for describing scattering with momentum transfers comparable with energy.

As a concrete example, we choose a quasipotential in the form of a Gaussian

$V(s, r)=i s\left(\frac{\pi}{a}\right)^{3 / 2} \exp \left(-r^{2} / 4 a\right)$

corresponding to a purely imaginary amplitude of diffraction scattering. In (1) the parameter $a$ characterizes the effective interaction radius, which depends on the energy. With increasing energy, the parameter $a$ increases logarithmically: $a=a_{0}+$ lns . The local quasipotential (1) has a positive definite imaginary part and is a smooth non-singular 
function of $r$ which satisfies all of the principles enumerated above and also the requirement of diffractive behaviour at small transferred momenta. The advantage of Gaussian Potential is that it is very flexible. In contrast to analytic potentials, the accuracy of Gaussian potential can be improved by adding more quantum mechanical data at various points in configurational space without changing the fit globally. The flexibility of the fit ensures that the best possible fit is achieved for any given data [7].

In the representation of invariant variables (Mandelstam variables) the standard relationship of the scattering amplitude $f$ with the differential cross section of elastic scattering has the following form

$\frac{d \sigma_{e l}(s, t)}{d t}=|f(s, t)|^{2}, \quad s=4\left(k^{2}+m^{2}\right)=4 E^{2}$,

$t=-(\mathbf{p}-\mathbf{k})^{2}$.

The scattering amplitude is analytic not only in the $s$ plane, but also in the $t$ plane.

In the description of elastic diffraction, it is convenient to use the eikonal model, the advantage lies in the fact that in an explicit form it leads to the observance of the unitarity condition for the scattering amplitude. If the energy of the incident particle is sufficiently large, so that the wavelength $\square=\kappa^{-1}$ is small in comparison with the characteristic dimensions of the interaction region $R$ (where $R$ is the nucleus radius), i.e. $k R \gg>1$, the so-called high-energy approximation is well-suited to explain the scattering of such particles.

The high-energy or eikonal approximation is widely and successfully used to describe the scattering of particles also in complex nuclei as scattering in a certain optical continuous medium. In this approximation, instead of the law of conservation of energy, the law of conservation of the momentum projection on the direction $\mathbf{k}$ takes place: $\mathbf{p k}=$ const. This means that the movement in the transverse directions is completely neglected. In addition, in this approximation, no restrictions are imposed on the masses and coordinates of the particles, both the finite radius and the recoil are considered exactly. Therefore, the eikonal approximation can be used to calculate the differential cross sections for both direct and exchange processes. In addition, in the eikonal approximation, the effect of distortions is taken into account only in the phase of a plane wave. Therefore, the high-energy approximation can be used to calculate the angular distributions of scattered particles.

For the hadron scattering amplitude at high energies, it is very convenient to pass from expansion in partial waves to the representation of the impact parameter $b$ :

$$
f(s, b)=i \int b d b\left(1-\exp \left[-\frac{i}{m} \int_{-\infty}^{z} V\left(b^{2}+z^{\prime 2}\right) d z^{\prime}\right]\right)
$$

Such a recording of the amplitude in the highenergy region is not based on a specific mechanism of interaction. The entire dynamics of the process in the eikonal models should be introduced by specifying a specific kind of eikonal [8]:

$\chi(s, b)=i\left(\exp \left(-\mu \sqrt{b^{2}+a^{2}}\right)-\exp \left(-2 \mu \sqrt{b^{2}+a^{2}}\right)\right)$

here the parameters $\mu_{0}$ and $a_{0}$ have the meaning of the reduced mass and the interaction radius:

$$
\begin{aligned}
& \mu=\mu_{0} / \sqrt{1+\ln s-i \pi / 2} \\
& a=a_{0} / \sqrt{1-\ln s-i \pi / 2}
\end{aligned}
$$

In the eikonal approximation, the characteristic scattering angle is determined by the quantity $\theta \leq \lambda / b$.

(3) can be expressed in terms of the eikonal

$$
f(s, t)=i \int b d b(1-\exp [i \chi(s, b)]) J_{0}(b \sqrt{-t})
$$

where $J_{0}$ is the Bessel function. 
We note that the function $f(s, b)$, which determines the amplitude for purely nuclear scattering according to Eq. (6), is virtually equal to unity within the nucleus, both in the case of antinucleon scattering, and in that of nucleon scattering. This means that for these particles the nucleus acts as an absolutely black sphere (in the central region) with a diffuse boundary.

At high energies, each value of $b$ corresponds to its partial wave $l=b \sqrt{s / 2}$ and the unitarity condition has the form

$$
2 \operatorname{Im} f(s, b)=|f(s, b)|^{2}+\varphi_{\text {in }}(s, b),
$$

here $\varphi_{\text {in }}(s, b)$ is the contribution of inelastic channels, i.e. probability of inelastic interaction at a point $b$.

The total collision cross section and the inelastic scattering cross section are determined as follows

$$
\begin{aligned}
& \sigma_{\text {tot }}=4 \pi \int \operatorname{Im} f(s, b) b d b \\
& \sigma_{\text {in }}=2 \pi \int \varphi_{\text {in }}(s, b) b d b
\end{aligned}
$$

The differential cross section for elastic scattering is related to the amplitude as follows

$$
\frac{d \sigma(s)}{d t}=\left[\operatorname{Re} f(s, t)^{2}+\operatorname{Im} f(s, t)^{2}\right]
$$

The elastic scattering amplitude must satisfy the general principles of analyticity and unitarity.

After simple calculations for the differential cross section for elastic scattering, we obtain

$$
\frac{d \sigma}{d t}=\left(\frac{d \sigma}{d t}\right)_{t=0} \exp \left\{2[a(t)-1] \ln \left(s / s_{0}\right)\right\}
$$

The differential cross sections of binary processes (in particular, the elastic pp scattering reactions), according to formula (10), are concentrated in a narrow region of transmitted momentum $|t|$, whose width decreases logarithmically with increasing energy. This phenomenon in elastic processes is usually called the reduction of the diffraction cone. Reduction of the cone of the angular distribution was observed experimentally in all binary reactions.

The total cross section is related to the imaginary part of the scattering amplitude according to the optical theorem.

$$
\sigma_{t o t}=4 \pi \int \operatorname{Im} f(s, b) b d b=\frac{\operatorname{Im} f(0)}{s}
$$

In the $b$ representation the total cross section increases with increasing $s$ as

$$
\sigma_{\text {tot }} \propto \ln ^{2} s \text {. }
$$

Due to the fact that with increasing energy the cross section grows in the logarithmic approximation, as $s$ increases, it is necessary to noted that the distance at which the collision probability is not yet small increases with increasing according to the law

$$
r(\ln s)=a \ln s+d-c \ln s
$$

where $a, d, c$ are constants. The cross section with amplitude $f(s, b)$ and with radius (13) is a disc with radius $r \propto \ln s$. Inside the $\operatorname{disk}(b<\mathrm{r}) \operatorname{Im} f \square 1$, and at the periphery of the disk (b>r) $f \propto \exp \left(-2 m_{\pi}(b-r)\right)$. This behaviour ensures the correct position of the nearest feature of the $\mathrm{t}$ channel $t=4 m^{2}$.

The amplitude can be expanded in a series $b$ containing only even powers of the regularity condition for the Fourier-Bessel transform of the function (6):

$$
f(s, t)=i s \sum_{n=1}^{\infty} \frac{\exp (a|t| n)}{n ! \cdot n}\left(-\frac{4 \pi^{2} \mu}{a}\right)^{n-1} .
$$




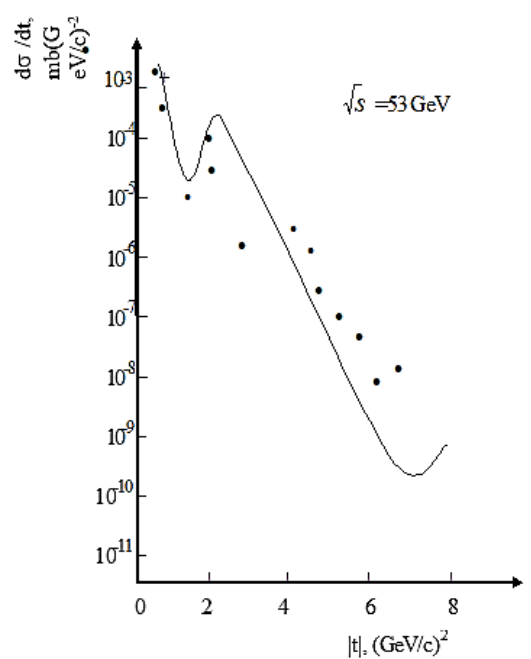

Figure 1. Dependence of the differential cross section of an elastic $p p$ scattering from. Points-experimental data from $[9,10]$.

On the basis of the obtained formulas, we first consider proton-proton scattering.

For $t \approx-\frac{2}{a} \ln \left(\frac{a}{\pi^{2}}\right)$ the contribution of the second term in the expansion (14) is comparable with the first term. Therefore, a second minimum must appear in the differential cross section. This fact is confirmed by the known experimental data $[9,10]$ for measuring the differential cross section for elastic $p p$ scattering at $\sqrt{s}=53 \mathrm{GeV}$. The parameters are adjusted to experimental data. As can be seen from Fig.1, the model predicts the first minimum at $\sim 1.2(\mathrm{GeV} / \mathrm{c})^{2}$ and the second minimum at $\sim 7.0(\mathrm{GeV} / \mathrm{c})^{2}$. There are three minimums for the cross section in the experiment. The first and third coincide with the theoretical values, but the second minimum does not exist on the theoretical curve. Undoubtedly, the imaginary part of the elastic scattering amplitude dominates at small angles in the diffraction cone and the problem of the behaviour of the real part of the amplitude of elastic scattering for nonzero momentum transfers becomes very relevant.
One might think that the discrepancy is due to the fact that at high energies because of intense meson formation, all phases become complex and the nuclear amplitude - forward scattering is almost completely imaginary. In this case, the scattering can be represented as due to the action of the onepion exchange potential. In the paper [11], protonproton elastic scattering was studied in the framework of one pion exchange model and a scalar theory in an attempt to simulate nucleonnucleon interactions covering a large energy range. When the models were compared with the available total cross-sectional data, it was found that the scalar theory best fits the data below the below labotary momentum of $0,5 \mathrm{GeV}$. However, compliance with high-energy data is not as good as it was found in a very low momentum region.

In Fig. 2 compares the results of calculations using formula (11) with experimental data for the scattering cross section $p^{9} \mathrm{Be}$ [12]. The solid line corresponds to the cross-section calculated from equation (11), the points correspond to the experimental data.

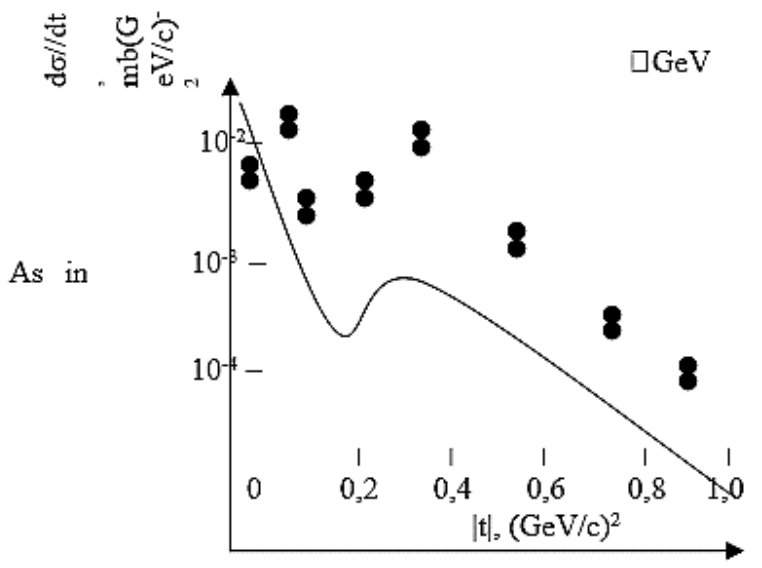

Figure2. Differential cross section for $p^{9} \mathrm{Be}$ scattering. The solid line corresponds to the cross-section calculated from equation (11), the points correspond to the experimental data from [12]. 
It can be seen from Fig. 2 that the model of a composite nucleon leads to a satisfactory agreement between the calculated cross section and its t-dependence and experimental data. As in the usual eikonal approach, at large $|\boldsymbol{t}|$ values are predicted $d \sigma / d t$ (Fig.1. and Fig.2) that are significantly smaller than in the experiment. In addition, there is no pronounced minimum in the experiment. The experimental cross section has a slight inflection at $|\mathrm{t}|=0.4(\mathrm{GeV} / \mathrm{c})^{2}$. The unitarity condition predicts an exponential fall for the differential cross section with additional substructure to occur exactly between the low momentum transfer diffraction cone and high momentum transfer. As can be seen from the Fig.2, the steep Gaussian peak at small angles is followed by the exponential (Orear) regime with some shoulders and dips and then by the power-law drop.

It is known that the ${ }^{9} \mathrm{Be}$ nucleus does not have spherical symmetry, and one of the reasons may be a significant deformation of the nucleus, which has a significant quadrupole moment $\mathrm{Q}=53 \mathrm{mb}$. We made very simple assumptions about the structure of the nuclei and indicated the main theoretical considerations for detailed calculations. Depending on the number of nucleons, the number of channels influencing the decay channel increases. If deformed nucleus, the deformation also affects the process. Other reasons are an increase in the ratio of the real part of the elementary scattering amplitude to the imaginary one and the presence of some incoherent scattering channels. Accounting for all of these factors sharply increases the complexity of the calculations. Therefore, this article did not take into account the role of deformation and number of channels.

It follows from (9) that the ratio of the real part of the amplitude in the forward scattering to its imaginary part

$$
A(s, t)=\frac{\operatorname{Im} f(s, t)}{\operatorname{Re} f(s, t)}
$$

must tend to zero in the asymptotic as $s$ increases. A definite relation between the real and imaginary parts of the amplitude is valid for a certain energy interval.

The dependence of the $A(s, t)$ on $t$ for $\mathrm{E}>150$ $\mathrm{GeV}$ is shown in Fig.3. The ratio $A(t)$ becomes zero for $|t|^{2} \approx 0,42$ and for large values of $t$ it tends to $\approx-9$. The result, shown in Fig.3, must be considered as follows from the unitarity condition. This decrease shows that it is more difficult for particles to dissipate with larger transverse momenta, preserving their integrity. As we can see, the real part of the amplitude can overexceed at large momentum transfers, according to the unitarity condition. In this region, the real part of the amplitude can be large and negative in comparison with its imaginary part. We note that $A(t)$ vanishes and becomes negative. This result agrees with the general theorem on the change in the sign of the real part of the elastic scattering amplitude at high energies [13]. But on the other hand, the decrease in $\boldsymbol{A}(t)$ with an increase in $t$ is unsatisfactory. $A(t)$ is very sharply decreasing in the region $0.4-0.7 \mathrm{GeV}^{2}$. More peripheral interactions with large $A(t)$ are characterized by small momentum transfers. The result given in Fig. 3 should be considered as one more extreme approximation to the true solution of the equation expressing the unitarity condition.

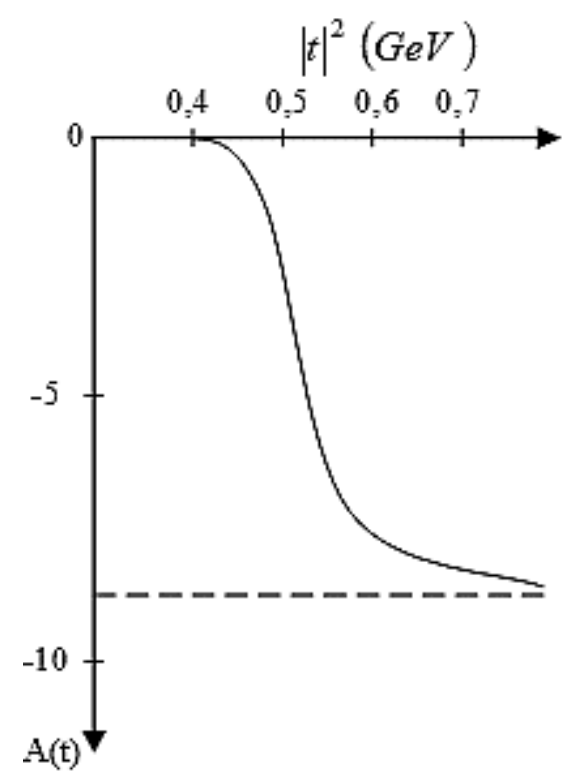

Figure 3. Dependence of the $A(\mathrm{t})$ of an $\left.|| \mathrm{t}\right|^{2}$. 
general theorem on the change in the sign of the real part of the elastic scattering amplitude at high energies [13]. But on the other hand, the decrease in $A(t)$ with an increase in $t$ is unsatisfactory. $A(t)$ is very sharply decreasing in the region 0.4 $0.7 \mathrm{GeV}^{2}$. More peripheral interactions with large $A(t)$ are characterized by small momentum transfers. The result given in Fig. 3 should be considered as one more extreme approximation to the true solution of the equation expressing the unitarity condition.

In explaining the energy dependence of the hadronhadron scattering cross section, the $t$-channel exchange picture is more adequate, which for high energies was formulated in the form of the Regge model. Diffraction scattering in the Regge model is described by the exchange of a pomeron-a vacuum moving pole in the complex plane of the angular momentum. But this model also can not explain all the data. $(\mathrm{s}, \mathrm{t})$-dependence of the differential cross sections and the ratio $A(\mathrm{t})$ in a wide energy range and momentum transfers can not be solved without introducing fitting parameters.

\section{CONSCLUSION}

In this paper we carried out a study on the basis of the continued unitarity method in which the main equation for the scattering amplitude is the elastic unitarity condition, analytically continued to the region above the first inelastic threshold. Here we considered only the Gaussian potential. Nevertheless, the physical interpretation of the results suggests [14], that the qualitative scattering pattern described in the main features is valid for a larger class of strong potentials that decrease rapidly at infinity.

We have made very simple assumptions regarding the structure of the nuclei and pointed out the main theoretical considerations for detailed calculations. More specific structure effects, such as e.g. resonances, are expected to appear on a background parameterized by the above equations. The availability of experimental data in the near future will certainly arouse interest on the detailed investigation of such effect. Such experiments would give valuable information on disintegration reactions and about the distribution of the nuclear density in the nuclear surface. At high energies both the electromagnetic and the nuclear interaction between projectile and target will be important. Far from being a drawback, this can be of utility to extract complementary information about these different reaction mechanisms in the peripheral collisions. A decomposition of these mechanisms from the analysis of angular distribution of the fragments or from the dependence of the cross sections on the energy, charge and mass parameters is possible in accurate measurements.

There are many models, but it is still difficult to give preference to any one of them. Most models are successful at the introduction of different assumptions. It is essential how to achieve improvement of existing models: by avoiding simplifying assumptions or looking for new physical arguments that would make the model more realistic and save it from simplifications. It is from this point of view that one should approach the analysis of the further development of the model of hadron scattering, caused by new experimental facts.

\section{REFERENCES}

[1]. C.A. Bertulani and G. Baur, N. Ph, 1988, A 480, 615- 628.

[2]. N. P. Zotov, V. A. Tsarev, Sov. Phys.Uspekhi, 1988, 31 (2), 119-139.

[3]. D. Kiang, S.H Ling, K.Young, C.S. Lam, Phys.Rev. D, 1985, 31-35.

[4]. A.A. Logunov and A.N. Tavkhelidze, Nuovo Cim. 1963, 29, (2), 380-399.

[5]. Yu.D. Chernichenko Physics of Atomic Nuclei, 2015, Vol. 78, (2), 201-214.

[6]. W. Ulmer. Jour Proton Ther. 2015;1:117. DOI: http://dx.doi.org/10.14319/jpt.11.7

[7]. Bartók-Pártay, DOI: 10.1007/978-3-64214067-9,7, Springer-Verlag Berlin Heidelberg, 2010, p. 83.

[8]. S.G. Abdulvahabova, E.A. Rasulov, Proceeding of International workshop "Quantum particles, fields and strings" Fizika, 2002, (3), 83- 85. 
[9]. H.S. Hans. Nuclear Physics: Experimental and Theoretical. New Age Pulisher, 2001, p.787

[10].N. P. Zotov, S.V. Rusakov, V. A. Tsarev. PEPAN, 1980, V.11(5), 1160-1223.

[11].Ryan B. Norman, Frank Dick, John W. Norbury and Steve R. Blattnig. Differential Cross Sections for Proton-Proton Elastic Scattering NASA/TP, 2009, 215565, p. 87.
[12].G.D. Alkhazov, S.L. Belostotsky, A.A. Vorobiev. Scattering of protons by light nuclei. Preprint LINP-449, 1978, p. 11.

[13].A. Martin, Phys. Rev. D, 2009, 80, 065013.

[14].I. M. Dremin, Uspekhi Fizicheskikh Nauk, 2013, 183 (1), 3-32. 